\title{
The function of complexes between the outer mitochondrial membrane pore (VDAC) and the adenine nucleotide translocase in regulation of energy metabolism and apoptosis
}

\author{
Mikhail Y. Vyssokikh ${ }^{1}$ and Dieter Brdiczka ${ }^{2}$ \\ ${ }^{1}$ A.N. Belozersky Institute of Physico-Chemical Biology, Moscow State University, Moscow, \\ Russia; ${ }^{2}$ Department of Biology, University of Konstanz, Konstanz, Germany
}

Received: 9 May, 2003; accepted: 28 May, 2003

Key words: hexokinase, creatine kinase, free energy, permeability transition pore, Bax, cytochrome $c$

\begin{abstract}
The outer mitochondrial membrane pore (VDAC) changes its structure either voltage-dependently in artificial membranes or physiologically by interaction with the adenine nucleotide translocase (ANT) in the c-conformation. This interaction creates contact sites and leads in addition to a specific organisation of cytochrome $c$ in the VDAC-ANT complexes. The VDAC structure that is specific for contact sites generates a signal at the surface for several proteins in the cytosol to bind with high capacity, such as hexokinase, glycerol kinase and Bax. If the VDAC binding site is not occupied by hexokinase, the VDAC-ANT complex has two critical qualities: firstly, Bax gets access to cytochrome $c$ and secondly the ANT is set in its c-conformation that easily changes conformation into an unspecific channel (uniporter) causing permeability transition. Activity of bound hexokinase protects against both, it hinders Bax binding and employs the ANT as anti-porter. The octamer of mitochondrial creatine kinase binds to VDAC from the inner surface of the outer membrane. This firstly restrains interaction between VDAC and ANT and secondly changes the VDAC structure into low affinity for hexokinase and Bax. Cytochrome $c$ in the creatine kinase complex will be differently organised, not accessible to Bax and the ANT is run as anti-porter by the active creatine kinase octamer. However, when, for example, free radicals cause dissociation of the octamer, VDAC interacts with the ANT with the same results as described above: Bax-dependent cytochrome $c$ release and risk of permeability transition pore opening.
\end{abstract}

Eukaryotic porins are membrane proteins that form aqueous channels in the cell mem- brane and the mitochondrial outer membrane. In contrast to bacterial porins that

\footnotetext{
${ }^{{ }^{\top}}$ To whom correspondence should be addressed: Department of Biology, University of Konstanz, D 78457 Konstanz, Germany; e-mail: Dieter.Brdiczka@uni-konstanz.de

Abbreviations: ANT, adenine nucleotide translocase; CK, creatine kinase; HK, hexokinase; VDAC, voltage-dependent anion channel.
} 
have a similar function, the structure of eukaryotic porins is not known at a useful resolution. The mitochondrial outer membrane pore was first characterised by Marco Colombini (1979) as a voltage dependent anion channel (VDAC). The properties of VDAC have been widely investigated in reconstituted systems by several groups (Colombini, 1979; Benz, 1994) exploring the main differences compared to bacterial porins. These are: sensitivity to voltage already at $30 \mathrm{mV}$ and to polarity of the applied voltage. In general, bacterial and mitochondrial outer membranes have segregating functions and pore proteins in the membranes control limited exchange. However, in bacteria the outer membrane has more protective functions, whereas in mitochondria communicative functions stand in the foreground.

VDAC plays an important role in coordination of communication. A substantial aspect of this management is a transient formation of complexes with other proteins. This will be the topic of the present article.

\section{STRUCTURE OF VDAC}

Although the VDAC amino-acid sequence is very different compared to the bacterial porins, it is assumed the mitochondrial outer membrane pore may form a $\beta$-barrel composed of $16 \beta$-strands analogous to the know structure in bacteria (Casadio et al., 2002). Three different isotypes of VDAC are expressed in cells (Sampson et al., 1997). The function of them will not be described in details. All experiments mentioned here relate to VDAC isotype I, which is the main species in the outer mitochondrial membrane.

\section{VDAC CONDUCTANCE AND ION SELECTIVITY}

Analysis of the isolated VDAC after reconstitution in artificial bilayer membranes re- sulted in the calculation of the pore diameter of $4 \mathrm{~nm}$ at a voltage smaller than $30 \mathrm{mV}$. In this high conductance state $(4 \mathrm{nS}$ at $1 \mathrm{M} \mathrm{KCl}$ in the bathing fluid) the pore is anion selective. Above $30 \mathrm{mV}$ the diameter of the pore is reduced to $2 \mathrm{~nm}$. The conductance decreases to $2 \mathrm{nS}$ and ion selectivity changes to cation-selectivity (Colombini, 1979; Benz, 1994). The voltage-dependent conductance variations are linked to large structural modification of VDAC. So far no information about the nature of this changes is available. It has been postulated that a positively charged loop moves out of the channel (Song et al., 1998). But it is also possible that negative charges move into the mouth of the channel as it has been observed for bacterial porins (Welte et al., 1995). Macromolecules such as dextran, that can not penetrate the pore, have an osmotic effect on the aqueous interior of the channel (Zimmerberg \& Parsegian, 1986) and by that increase voltage sensitivity. In the presence of dextran the low conductance cation selective state is adopted already at $10 \mathrm{mV}$ (Gellerich et al., 1993). It has been postulated that the pore acquires this state in the contact sites where the inner membrane potential extends to the outer membrane by capacitative coupling (Benz et al., 1990). Recently a new idea of generation of a potential at the outer membrane was proposed on a basis of metabolic cycling and translocation of ATP, ADP, and $P_{i}$ across the inner membrane and different resistance in the contact sites (Lemeshko, 2002).

\section{PHYSIOLOGICAL SIGNIFICANCE OF THE VOLTAGE DEPENDENCE}

VDAC in the low conductance, cation-selective state is not permeable for ATP, ADP and other negatively charged small molecules. This has been demonstrated by Colombini and co-worker investigating VDAC reconstituted in artificial membranes (Rostovtseva \& Colombini, 1997). In isolated mitochondria, 
enzymes in the inter-membrane space, such as adenylate kinase, have no access to external adenine nucleotides in the presence of the König's polyanion (König et al., 1977) that induces the low conductance state of VDAC (Benz et al., 1988). If we convey this results to the physiological situation where more contact sites are formed and macromolecules are present, we have to assume that most VDACs are in the low conductance cation selective state. Indeed, mitochondria in situ of permeabilised cardiomyocytes or skinned red muscle fibres have a 10 times higher apparent $K_{\mathrm{m}}$ for ADP compared to isolated mitochondria from the same tissue. The high $K_{\mathrm{m}}$ could be reduced to normal values by generating porous outer membranes with digitonin or by the pro-apoptotic protein Bax suggesting that in situ the outer membrane was not freely permeable for ADP (Saks et al., 1995). Besides pure voltage dependent regulation, specific proteins in the intermembrane space or attached to the surface have been described that physiologically may also be involved in regulation of the pore permeability (Liu \& Colombini, 1992; Saks et al., 2001).

\section{PORINS AS SPECIFIC BINDING SITES}

In bacteria porins are receptors for various phages. VDAC at the mitochondrial periphery acts in a similar way as binding site for enzymes such as hexokinase (Fiek et al., 1982) and glycerol kinase (Östlund et al., 1983). A shift in transmembrane topology in the membrane also changes the surface-exposed loops between the $\beta$ strands. Considering the voltage dependence of the transmembrane topology, this means that VDAC in the contact sites might have the role to reflect functional changes of the inner membrane potential at the mitochondrial surface. Alternatively, such signalling to the surface of the outer membrane may be generated by interaction of VDAC with the ANT (see below) if we assume that the ANT would interact exclusively with a certain VDAC state.

\section{CONTACT SITE CHARACTERISATION AND ISOLATION}

In support of this idea, it has been observed by immune electron microscopy and binding studies that hexokinase binds with higher capacity to the outer membrane pore in the contact sites compared to pores beyond contacts (Weiler et al., 1985). Freeze fracturing analysis of isolated liver mitochondria revealed that contact sites could be induced by dextran or atractyloside and suppressed by glycerol or DNP (Wicker et al., 1993; Bücheler et al., 1991). Thus, hexokinase binding to isolated outer membrane or mitochondria in state of induced or suppressed contact sites was studied. Hexokinase showed sigmoidal type of binding to mitochondria with contact sites, while binding to pure outer membrane or mitochondria with suppressed contact sites led to hyperbolic binding curves (Wicker et al., 1993). This suggested a co-operative binding of hexokinase to the pore in the contact site area. In agreement with this, hexokinase, by binding, was activated and bound hexokinase was found to form tetramers (Xie \& Wilson, 1990). In a recent investigation Hashimoto and Wilson (2000) were analysing different epitops at the surface of bound hexokinase by specific monoclonal antibodies. The authors observed a variation of the bound hexokinase structure that correlated with functional changes of oxidative phosphorylation in the inner membrane. This direct reflection of inner membrane functions at the mitochondrial periphery can be explained by the interactions between VDAC in the outer and ANT in the inner mitochondrial membranes (Bühler et al., 1998; Beutner et al., 1996; Vyssokikh et al., 2001).

Because of the high capacity of contact sites for hexokinase binding, the enzyme was used as a marker to isolate this membrane fraction 
from osmotically disrupted mitochondria by density gradient centrifugation (Ohlendieck $e t$ al., 1986; Adams et al., 1989). Recent application of the contact site isolation revealed in kidney mitochondria that hexokinase activity
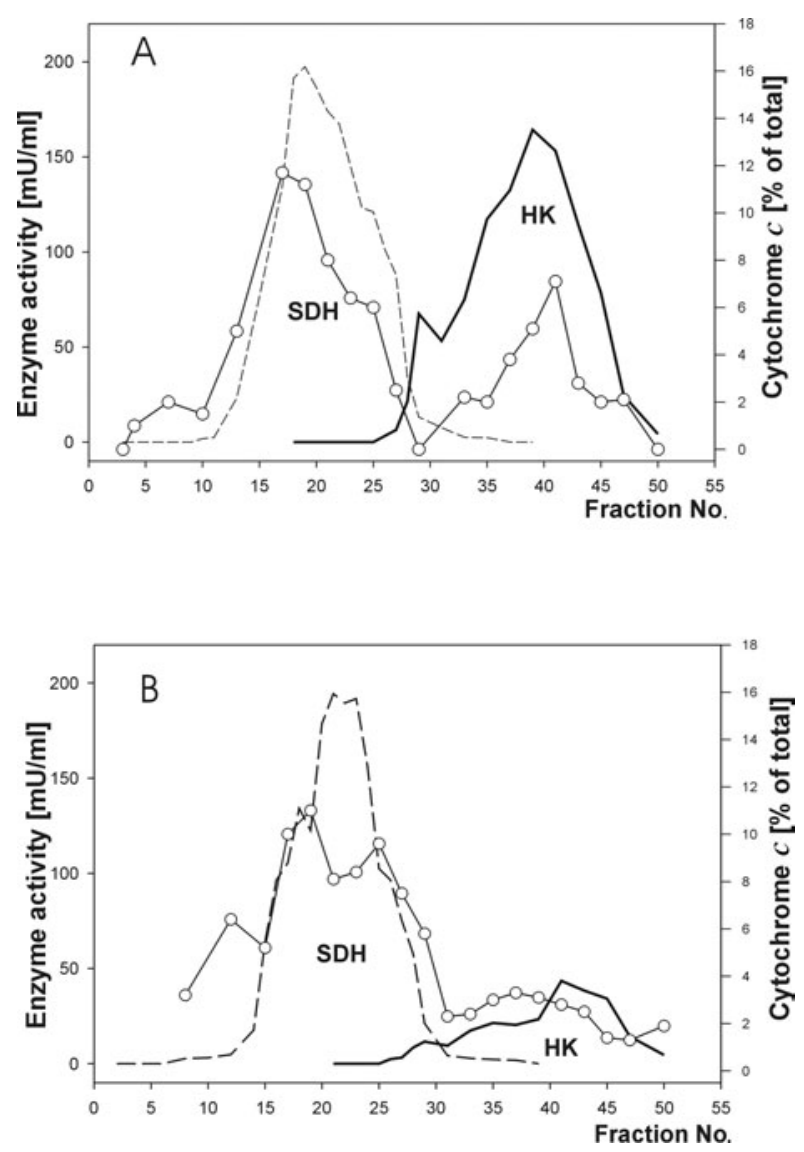

Figure 1. Variation of cytochrome $c$ and hexokinase in the contact site fraction of kidney mitochondria by perturbing porin-ANT complexes.

Distribution of inner membrane and contact site fractions in a sucrose density gradient separating sub-fractions of rat kidney mitochondria after osmotic shock. The gradient was divided into 55 fractions (Fraction No.) starting from $54.3 \%$ sucrose, left to $30.8 \%$, right. Succinate dehydrogenase (SDH, dashed line) and hexokinase (HK, solid bold line) were used as markers for the inner and the outer membranes, respectively. Cytochrome $c$ concentration (empty circles) in the different fractions was determined by specific antibodies and varied significantly in $500 \mu \mathrm{M}$ atractylosidetreated (panel A), compared to $250 \mu \mathrm{M}$ bongkrekatetreated (panel B) mitochondria. in the contacts was decreasing when the ANT was shifted to the m-conformation by pretreatment with bongkrekic acid, whereas it was increasing after induction of the c-conformation by pre-incubation with atractyloside (Fig. 1).

We do not know how the structure of the VDAC changes and whether the alterations are induced by the membrane potential or by the interaction with the ANT. However, we can summarise the observations of voltage-dependent conductance changes and studies of hexokinase binding as showing that VDAC alters its transmembrane topology correlated to functional mitochondrial states. Because it affects the affinity and capacity of hexokinase binding, we can define a tensed and relaxed state of the pore in analogy to the regulation of allosteric enzymes. The tensed state would be the low conductance pore in a complex with the ANT, whereas the relaxed state would be characterised by high conductance without complex formation (Fig. 2).

\section{CYTOCHROME $c$ IS A COMPONENT OF THE CONTACT SITES}

It was observed that not only hexokinase but also cytochrome $c$ was a component of the contact sites (Więckowski et al., 2001) and its concentration increased in the presence of atractyloside and decreased upon treatment with bongkrekic acid (Fig. 1). Thus, similarly as observed for hexokinase, the structure of the ANT was responsible for the cytochrome $c$ distribution at the surface of the inner membrane.

\section{ISOLATION AND CHARACTERISATION OF VDAC-ANT COMPLEXES}

Complexes between VDAC and ANT were generated in vitro with isolated porin and ANT proteins (Bühler et al., 1998). But they were also isolated from brain or kidney mem- 
branes, solubilized in Triton X-100, by binding the attached hexokinase to an anion exchanger. It was found that the bound hexokinase complex contained VDAC and ANT isotype I (Vyssokikh et al., 2001). From the same membrane extracts a second complex composed of porin, creatine kinase, and ANT could be isolated (Beutner et al., 1996; Beutner et al., 1998). Based on the separation and purification of the different complexes two types of VDAC-ANT aggregates could be defined: one complex where VDAC and ANT interacted directly and VDAC achieved higher affinity for hexokinase (Fig. 2) and a second complex where VDAC interacted indirectly with the ANT through mitochondrial creatine present (Beutner et al., 1996). Hexokinase is active as a monomer of molecular mass 100 $\mathrm{kDa}$, whereas the functionally active mitochondrial creatine kinase unit is a dimer with molecular mass $85 \mathrm{kDa}$. Four dimers of mitochondrial creatine kinase form a cubic structure with identical top and bottom faces that were able to connect two membranes (Rojo et al., 1991). It is known from in vitro studies that the octamer of mitochondrial creatine kinase interacts directly with the outer membrane pore (Brdiczka et al., 1994), while the interaction with the ANT may be indirect through cardiolipin (Schlattner et al., 2001) that is known to be tightly bound to the ANT (Beyer \& Klingenberg, 1985) (Fig. 2).

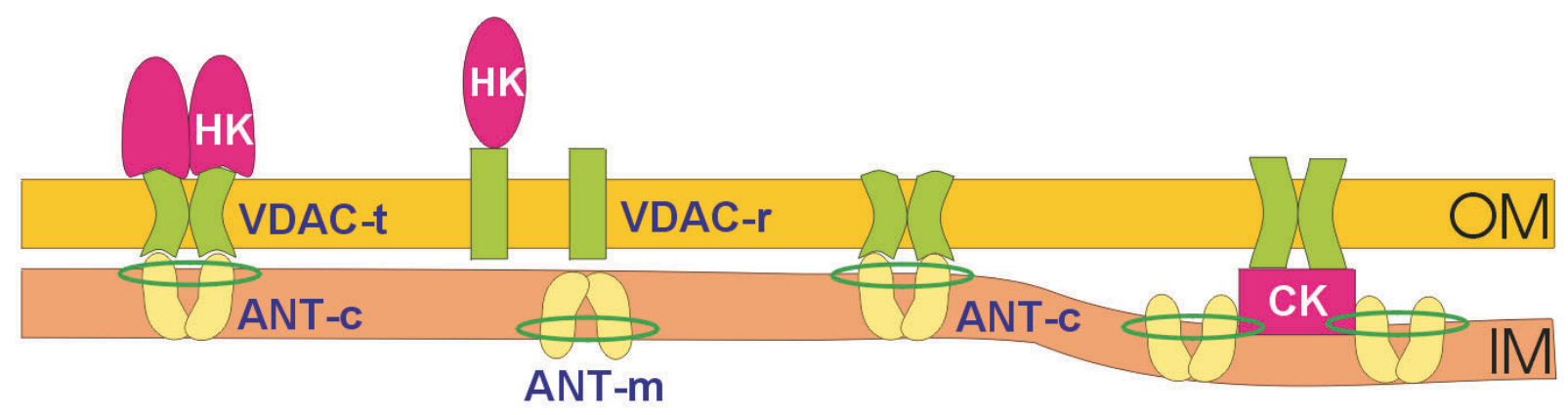

Figure 2. Different configurations of the VDAC-ANT complex connected to either hexokinase or creatine kinase.

Each of the three components of the two kinase complexes can exist in different configurations that support or inhibit complex formation. The adenine nucleotide translocator (ANT-c, induced by atractyloside) is facing the cytosol and interacts with the outer membrane pore (VDAC), whereas ANT-m (induced by bongkrekate) is orientated to the matrix and does not interact with VDAC. The green ring represents cardiolipin that might change from outer to inner leaflet of the membrane correlated to the ANT structure. Active ANT is a dimer. VDAC-t (tensed) is a dimer when it interacts with the ANT-c. VDAC-r (relaxed) is not bound to ANT and may also exist as monomer. A third structure of VDAC, unable to bind hexokinase, is present in the complex with the octamer of creatine kinase (CK). Hexokinase (HK) associates to oligomers preferentially in the contact sites formed by the VDAC-ANT-c complex. The oligomerisation of hexokinase leads to activation of the enzyme. Monomers of hexokinase bind to VDAC beyond the contacts with less affinity and activity. Creatine kinase (CK) binds to VDAC as octamer. The octamer preferentially associates with cardiolipin that is bound to ANT.

kinase octamer. In this case VDAC exposed a different structure at the surface that had low affinity for hexokinase (Fig. 2) as hexokinase activity was always absent in the creatine kinase complexes.

Determination of the molecular mass of the isolated complexes suggested that in both cases tetramers of the active kinases were

\section{RECONSTITUTION OF VDAC-ANT COMPLEXES}

The hexokinase-porin-ANT complex and the porin-creatine kinase-ANT complex were isolated and functionally reconstituted in vesicles (Beutner et al., 1996). ATP loaded into the vesicles did not leak out, although VDAC was a 
component of the complexes. However, the kinases in the complexes had access to the internal ATP through the ANT. This was shown by either glucose-6-phosphate formation from external glucose via hexokinase or creatine phosphate generation by creatine kinase from external creatine. Both reactions were inhibited by atractyloside, a specific inhibitor of the ANT. The results suggested that the kinases, after reconstitution, were functionally coupled to the ANT. The ANT was controlling the exchange of adenine nucleotides through porin and was working as anti-porter (Krämer \& Palmieri, 1989). However, the functional state of the ANT in the reconstituted complexes could be converted into an unspecific uni-porter (resembling the permeability transition pore) by addition of $\mathrm{Ca}^{2+}$ as has also been demonstrated for several related mitochondrial transport systems by Dierks et al. (1990).

\section{IMPORTANCE OF METABOLIC CHANNELLING IN REGULATION OF ENERGY METABOLISM}

The tight functional coupling of peripheral kinases to the ANT is important because of two reasons: firstly, it regulates the activity of oxidative phosphorylation by $\mathrm{ADP}$, and secondly, it increases the free energy $(\Delta G)$ in the ATP system. To explicate the first point we may imagine the situation after eating a carbohydrate-rich meal: High glucose concentration enters the resting muscle cell that must be phosphorylated and converted to UDP-glucose to accomplish incorporation into glycogen. The ATP for this process is more efficiently provided by oxidation of pyruvate in the mitochondria than by lactate formation. Hexokinase bound to the VDAC-ANT complex induces the oxidative pathway by converting intra-mitochondrial ATP into ADP and by that it activates oxidative phosphorylation. To describe the second advantage of metabolic channelling, we have to consider that all ATP energy-consuming processes in the cell (including ion pumps) depends on the transfer of the $\gamma$ phosphoryl group of ATP according to the reaction: ATP $+\mathrm{X}=\mathrm{X}-\mathrm{P}+\mathrm{ADP}$. In a subsequent step X-P dissociates when, for example, ions are pumped. This means that the efficiency of ATP to drive these phosphorylation reactions depends on the level of ADP and inorganic phosphate $\left(\mathrm{P}_{\mathrm{i}}\right)$ during the reaction according to the equation of $\Delta G=\Delta G_{0}{ }^{\prime}+\mathrm{RT} \ln$ ([ADP] $\left.\left[\mathrm{P}_{\mathrm{i}}\right] /[\mathrm{ATP}]\right)$. Thus, the power $(\Delta G)$ of ATP and the flux through the ATP-dependent reaction would increase, if ADP and $\mathrm{P}_{\mathrm{i}}$ would be low or be continuously withdrawn from the reaction.

In the complex between mitochondrial creatine kinase and the ANT these requirements are perfectly fulfilled. The equilibrium of the reaction will not be reached, as ATP, just exported, is directly utilised and ADP produced is immediately taken up into the matrix by the ANT while $\mathrm{P}_{\mathrm{i}}$ is excluded by complex formation. Thus, metabolic channelling through functional coupling between ANT and creatine kinase pushes the balance of the reaction between creatine and ATP far to the side of phosphocreatine production. A further advantage of this coupling to the ANT is that the higher free energy of ATP at the surface of the inner membrane is preserved in appearance of a higher phosphocreatine/creatine quotient. ATP is electrogenically exported by the ANT at the expense of the membrane potential. It has been calculated that this process increases the free energy of ATP by additional $12 \mathrm{~kJ} / \mathrm{mol}$ (Heldt et al., 1972). Thus the phosphocreatine/creatine ratio could be increased by this quantity through coupling between mitochondrial creatine kinase and the ANT.

\section{THE VDAC-ANT COMPLEX AS PERMEABILITY TRANSITION PORE}

Mitochondria contain an unidentified structure that forms a large unspecific pore under conditions of high matrix $\mathrm{Ca}^{2+}, \mathrm{P}_{\mathrm{i}}$ and oxidative stress (Haworth \& Hunter, 1980). Opening of this so called permeability transition 
pore was fully reversible upon withdrawal of $\mathrm{Ca}^{2+}$ (Crompton \& Costi, 1988). Electrophysiological measurements revealed properties of the pore related to VDAC, suggesting that it may be a component of the whole structure (Zoratti \& Szabó, 1995; Kinally et al., 1993). The permeability transition pore could be specifically blocked by cyclosporin $\mathrm{A}$, which binds to cyclophilin $\mathrm{D}$, a peptidyl-prolyl-cis-trans isomerase present in the mitochondrial matrix (Crompton \& Costi, 1988; Halestrap \& Davidson, 1990). By employing a cyclophilin D affinity matrix, a complex between VDAC and ANT was isolated by Crompton et al. (1998) after extracting heart mitochondrial membranes with the detergent Chaps. This complex was reconstituted in vesicles and pore opening by $\mathrm{Ca}^{2+}$ and $\mathrm{P}_{\mathrm{i}}$ addition was registered in cyclosporin A-sensitive manner. Comparable results were obtained with the isolated hexokinase-porin-ANT complex. Cyclophilin was co-purified through isolation of the complex (Beutner et al., 1998). The complex was reconstituted in phospholipid vesicles that were loaded with malate. The internal malate was released, correlated to increasing $\mathrm{Ca}^{2+}$ concentrations. The process was inhibited by cyclosporin A, suggesting that the reconstituted hexokinase-porin-ANT complex had properties of the permeability transition pore (Fig. 3A).

Two questions are still discussed: firstly, whether ANT is the only structure that causes permeability transition, and secondly, whether ANT forms the pore only as a complex with VDAC. Concerning the first question, it is possible that all related antiporters such as $\mathrm{P}_{\mathrm{i}} / \mathrm{OH}^{-}$or Glut/Asp exchangers can form a permeability transition pore depending on the actual condition. As to the second question, it is possible to claim that also the purified ANT forms unspecific pores in the presence of $\mathrm{Ca}^{2+}$. This was shown by reconstitution of ANT in bilayer membranes (Brustovetsky \& Klingenberg, 1996) or in vesicles (Rück et al., 1998). Yet these pores were not sensitive to cyclosporin A, as cyclophilin D was missing.
Cyclophilin D is thus a second important regulatory component of the permeability transition pore (Crompton et al. 1988; Halestrap \& Davidson, 1990). The idea that VDAC may have as well regulatory functions was supported by the analysis of the reconstituted porin-creatine kinase-ANT complex. In contrast to the reconstituted hexokinase complex, there was no release of enclosed malate from the vesicles by addition of $\mathrm{Ca}^{2+}$ (Fig. 3B). As shown schematically in Fig. 3B, VDAC is linked to the octamer of creatine kinase that hinders interaction with the ANT. However, direct interaction between VDAC and ANT was possible after dissociation of the creatine kinase octamer. In this case malate was liberated dependent on $\mathrm{Ca}^{2+}$ addition (Fig. 3B). The release was inhibited by cyclosporin A. The results suggested that VDAC, similarly as cyclophilin D, might regulate of the permeability transition pore. A possible role of VDAC might be to keep the ANT in the c-conformation, exposing the ATP/ADP binding site to the cytosolic surface of the inner membrane according to the re-orientating carrier model proposed by Klingenberg (Klingenberg et al., 1971). In this conformation, that is induced by atractyloside, the conversion of the ANT into an unspecific pore by $\mathrm{Ca}^{2+}$ is facilitated and leads to permeability transition. However, if VDAC and ANT interact with the octamer of mitochondrial creatine kinase, conversion of ANT to the permeability transition pore-like state would be suppressed (Fig. 3B). In fact, this has been observed in a transgenic mouse model expressing mitochondrial creatine kinase in liver mitochondria (O'Gorman et al., 1997; Dolder et al., 2003). The permeability transition pore was opened in isolated mouse liver mitochondria by atractyloside and $\mathrm{Ca}^{2+}$. In contrast, in liver mitochondria from transgenic mice, containing creatine kinase, the permeability transition was inhibited by substrates that stabilised the octamer of creatine kinase, such as creatine and cyclocreatine. 

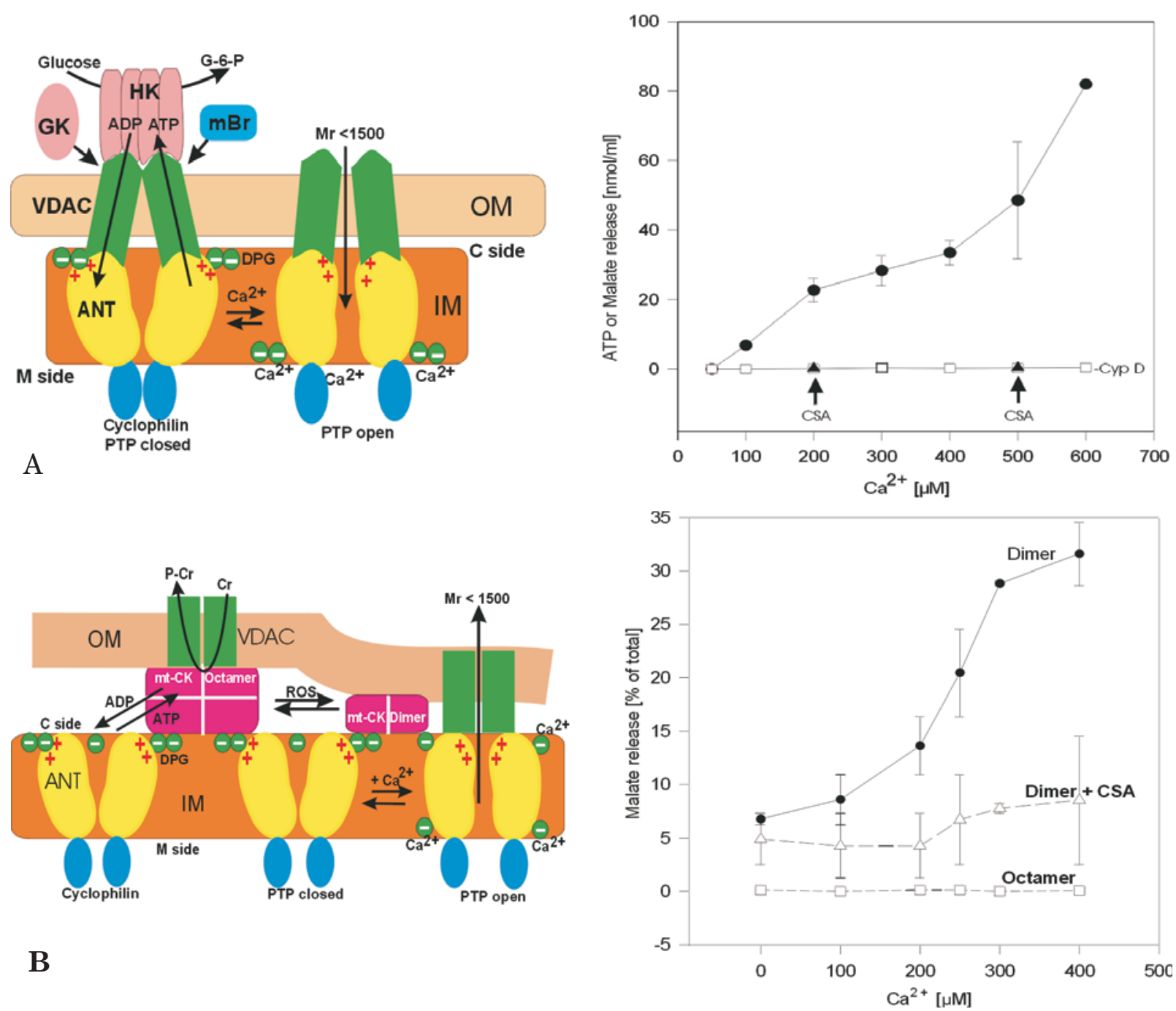

Figure 3. The VDAC-ANT complex as permeability transition pore.

A, left panel: The scheme depicts the presumable arrangement of the hexokinase-porin-ANT complex in the contact sites of mitochondria. Hexokinase (HK) binds as a tetramer to the VDAC-ANT complex. Glycerol kinase (GK) and the mitochondrial benzodiazepin receptor $(\mathrm{mBr})$ bind to the same pore configuration. A, right panel: The complex was isolated from a Triton X-100 extract of brain membranes and was reconstituted in phospholipid vesicles that were loaded with malate or ATP. Cyclophilin was co-purified with the complex and could be extracted from the complex with phosphate buffer, $\mathrm{pH}$ 4.5. The entrapped malate was released from the vesicles by $\mathrm{Ca}^{2+}$ between 100 and $600 \mu \mathrm{M}$. Malate release was inhibited either after cyclophilin extraction (-Cyp D) or by incubation of the vesicles with $0.5 \mu \mathrm{M}$ cyclosporin A (CSA) when 200 or $500 \mu \mathrm{M} \mathrm{Ca}^{2+}$ were added. According to Brustovetsky \& Klingenberg (1996) $\mathrm{Ca}^{2+}$ interacts with cardiolipin (DPG) around the ANT, thus changing the structure of the ANT from ATP/ADP antiporter to unspecific uniporter state. The uniporter state of ANT may act as permeability transition pore (PTP) in intact mitochondria. B, left panel: The scheme shows a possible arrangement of the complex between VDAC, the octamer of mitochondrial creatine kinase and the ANT in the contact sites of mitochondria. $\mathbf{B}$, right panel: The complex was isolated from the Triton X-100 extract of brain membranes and was reconstituted in egg yolk liposomes that were loaded with malate. Malate was not released by increasing $\mathrm{Ca}^{2+}$ concentrations (Octamer). When the octamer was dissociated into dimer by 20 min incubation of the liposomes with $5 \mathrm{mM} \mathrm{MgCl} 2$, $20 \mathrm{mM}$ creatine, $50 \mathrm{mM} \mathrm{KNO}_{3}$ and $4 \mathrm{mM} \mathrm{ADP}$, porin-ANT complexes could be formed. In these complexes $\mathrm{Ca}^{2+}$ could shift the ANT structure to the unspecific PTP and the entrapped malate was liberated (Dimer). Malate permeability of PTP was inhibited by pre-incubation of the vesicles with $100 \mathrm{nM}$ cyclosporin A (dimer + CSA). The octamer-dimer equilibrium of the mitochondrial creatine kinase can be shifted to dimer by reactive oxygen species (ROS) (Dolder et al., 2001). 
It should be mentioned here that mitochondrial creatine kinase, besides its important role in organisation of the peripheral mitochondrial compartment, is also localised in the cristae (Kottke et al., 1994) where it has certainly different functions and is not linked to VDAC.

\section{THE VDAC-ANT COMPLEX AS A TARGET FOR Bax DEPENDENT CYTOCHROME $c$ RELEASE}

There are several signal- and tissue-specific pathways to induce apoptosis. One of them is a release of cytochrome $c$ from mitochondria (Yang et al., 1997; Liu et al., 1996; Kluck et al., 1997) that activates caspases (Liu et al., 1996) by binding to a cytoplasmic protein Apaf-1 in presence of dATP (Saleh et al., 1999). The mechanism by which external Bax releases cytochrome $c$ is still controversial and may also depend on the actual localisation of cytochrome $c$ besides the binding and incorporation of Bax at the mitochondrial surface.

VDAC and ANT interact preferentially when adopting a specific molecular conformation. The ANT has to be in the c-conformation, as atractyloside induces the complex with the outer membrane pore (Vyssokikh et al., 2001), while bongkrekate shifts the ANT to the m-conformation and by that represses this interaction. VDAC in the complex with the ANT appears to be in the low conductance cationically selective, tensed state. This can be deduced from the observation that heart muscle mitochondria in situ show a reduced response to external ADP (Saks et al., 1995). The structural change of the outer membrane pore achieved by interaction with the ANT is recognised at the mitochondrial surface and leads to higher affinity of hexokinase. Recent observations suggest that this tensed pore structure is the preferred target of Bax molecules. Pastorino et al. (2002) found that hexokinase and Bax compete for the same binding site and
Capano and Crompton (2002) co-precipitated VDAC and ANT when Bax was immuno-precipitated from extracts of cardiomyocytes.

\section{THE VDAC-ANT COMPLEXES CONTAIN CYTOCHROME $c$}

As described above, it was observed that the contact sites contained cytochrome $c$. As the VDAC-ANT complexes are derived from the contact sites, it was not surprising that the complexes contained a significant amount of cytochrome $c$ (Więckowski et al., 2001; Vyssokikh et al., 2002). This was found, although the complex was eluted from anion exchanger column by $200 \mathrm{mM} \mathrm{KCl}$, suggesting that cytochrome $c$ was not bound by ionic interaction. To investigate whether Bax would be able to interact with the cytochrome $c$ within the VDAC-ANT complexes, the latter were reconstituted in phospholipid vesicles as described above (Fig. 4A). After reconstitution, the vesicles were loaded with malate. Bax liberated the endogenous cytochrome $c$ but did not release the internal malate (Fig. 4A). The Bax-dependent liberation of endogenous cytochrome $c$ was abolished when the VDAC-ANT complex was dissociated by bongkrekate (Fig. 4B).

\section{THE IMPORTANCE OF KINASES IN REGULATION OF APOPTOSIS}

Release of cytochrome $c$ from mitochondria proceeds in two steps: the first one involves the release of a small fraction of cytochrome $c$ from the compartment between the outer and the peripheral inner membranes and the contact sites. It is induced by Bax at low concentrations (Pastorino et al., 1999) in the early phase (Capano \& Crompton, 2002). The second step includes release of additional fractions of cytochrome $c$ located at the surface of the cristae membranes and results from opening of the permeability transition pore, fol- 

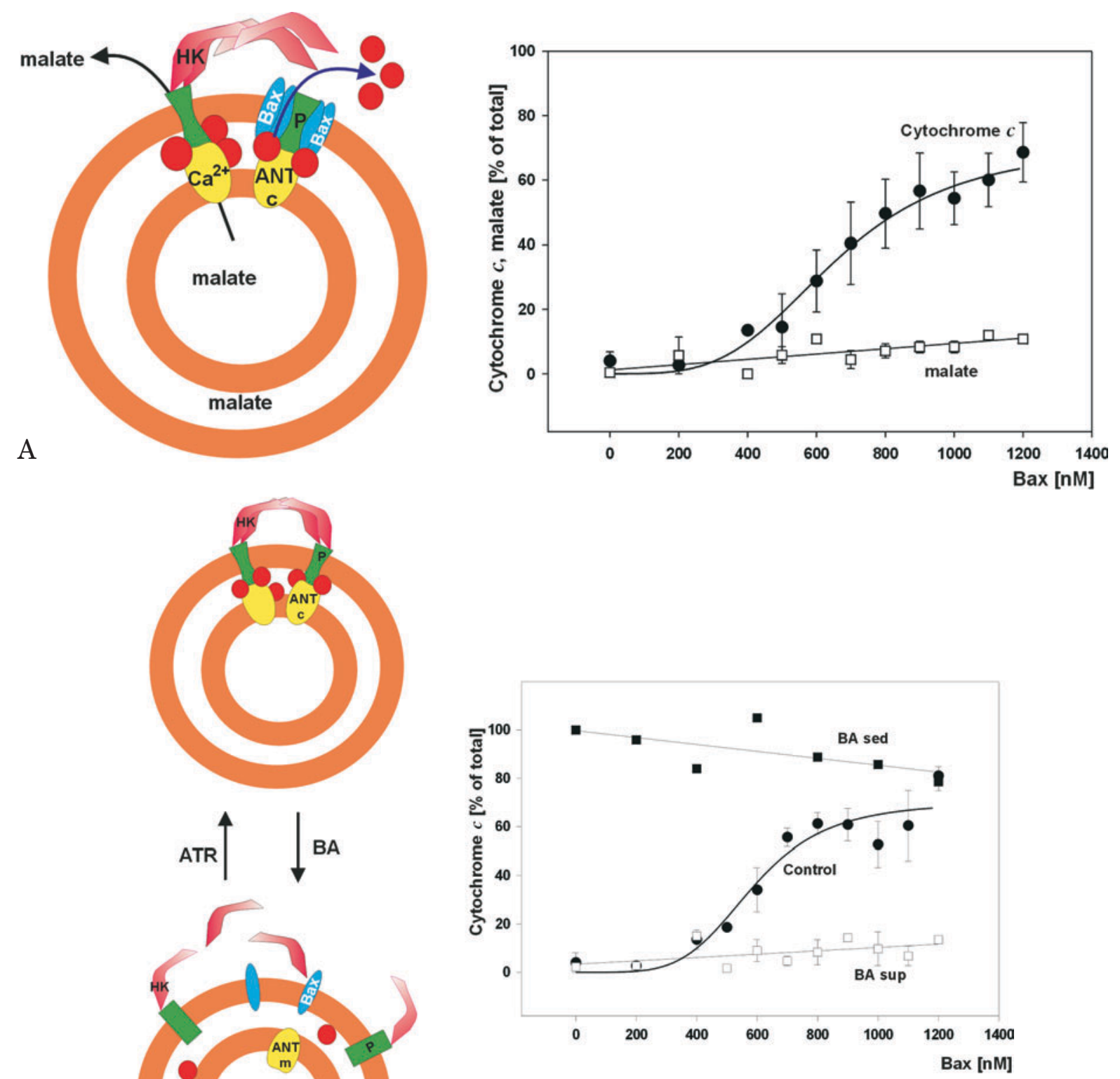

B

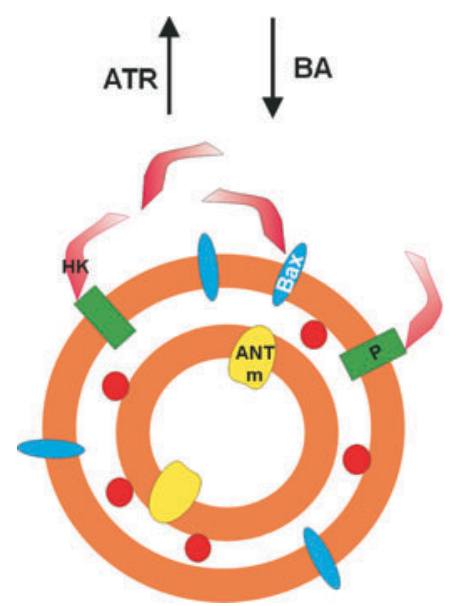

Figure 4. Bax-dependent release of cytochrome $c$ from the hexokinase-VDAC-ANT complex.

A, left side, schematic representation of the reconstituted hexokinase(HK)-VDAC(P)-ANT complex in multi-layer vesicles. Cytochrome $c$ (red circles), that was still attached to the complexes, could be released by Bax as shown on the right side. However, malate loaded into the vesicles was not liberated by Bax but by $200-500 \mu \mathrm{M} \mathrm{Ca}^{2+}$ through opening of ANT as permeability transition pore (Fig. 3A). This suggested that Bax specifically interacted with cytochrome $c$ in the porin-ANT complex. The sigmoidal curve of cytochrome $c$ release might indicate that Bax is accomplishing this process as oligomer. B, left side, schematic representation of the association/dissociation of the hexokinase(HK)-VDAC(P)-ANT complex by atractyloside (ATR) or bongkrekate (BA) after reconstitution in multi-layer vesicles. Treatment of the complexes with bongkrekate changed ANT into the m-conformation (ANT-m). This led to dissociation of the complex and a correlated structural change of VDAC, with decreasing affinity for hexokinase and Bax. Cytochrome $c$ might have redistributed as well. As shown on the right side, Bax was unable to release cytochrome $c$ after the structural change of the complex by bongkrekate. All cytochrome $c$ remained in the sediment (BA sed) in contrast to the control, without bongkrekate treatment, whereas no cytochrome $c$ appeared in the supernatant (BA sup). 
lowed by mitochondrial swelling and membrane disruption (Halestrap et al., 2002). The massive cytochrome $c$ release can also be a consequence of VDAC closure preceding membrane disruption (Vander Heiden et $a l .$, 1997). The observed failure of mitochondria to exchange adenine nucleotides with the cytosol may be a consequence of the transformation of VDAC into the tensed state. The two described kinases bound to VDAC are involved in the regulation of both processes of cytochrome $c$ liberation.

\section{SUPPRESSION OF Bax-DEPENDENT CYTOCHROME $c$ RELEASE AND PERMEABILITY TRANSITION BY HEXOKINASE}

Hexokinase binds to the outer membrane pore in its tensed state, when VDAC interacts with the ANT. In this state VDAC is not freely permeable for adenine nucleotides. This has been already observed in mitochondria in the presence of dextran that increases hexokinase binding and frequency of contacts between VDAC and ANT (Wicker et al., 1993). In these mitochondria external pyruvate kinase had less access to ADP produced by hexokinase (Gellerich et al., 2002). Although VDAC in the tensed state does not allow ATP transfer, hexokinase utilises mitochondrial ATP, suggesting a structural modification of VDAC through binding of the enzyme.

On the other side, the activity of mitochondrial bound hexokinase was found to be important for protein kinase B-linked suppression of cytochrome $c$ release and apoptosis (Gottlob et al., 2001). The experiments suggested that Bax, following withdraw of growth hormone, liberated a small fraction of cytochrome $c$ from the contact sites. In complete agreement with this, the Bax-dependent release of cytochrome $c$ bound in the VDACANT complexes described above was inhibited by activity of hexokinase in the presence of glucose and ATP (Vyssokikh et al., 2002). This was explained by stabilisation of hexokinase binding to VDAC that is also the main target of Bax. Besides this effect on Bax dependent cytochrome $c$ release, activation of hexokinase inhibited $\mathrm{Ca}^{2+}$-dependent opening of the permeability transition pore by ADP production (Beutner et al., 1998).

\section{SUPPRESSION OF THE PERMEABILITY TRANSITION BY MITOCHONDRIAL CREATINE KINASE}

Another kinase, which binds to VDAC, is mitochondrial creatine kinase. This enzyme interacts with porin exclusively in the octameric state, while the dimer has only weak affinity to porin (Schlattner et al., 2001). Thus the association-dissociation equilibrium between octamer and dimer determines the formation of octamer-VDAC complexes. Ligation of the coronary artery in guinea pigs led to decrease of octamer from $85 \%$ to $60 \%$ in the infarcted area of heart (Soboll et al., 1999). Because of the dissociation of the creatine kinase octamer, the possibility of direct VDAC-ANT interaction was increased. As suggested above, the latter complexes may be the prerequisite of $\mathrm{Ca}^{2+}$-induced permeability transition, followed by mitochondrial swelling, membrane disruption, cytochrome $c$ release and apoptosis. Indeed, apoptotic cells encircle the area of necrotic cells in infarcted heart. Heart, kidney and brain mitochondria contain two ANT isotypes. ANT isotype I is localised in the peripheral inner membrane, having higher affinity to VDAC and cyclophilin, whereas isotype II is present in the cristae membranes (Vyssokikh et al., 2001). When ANT I was over-expressed in cells, apoptosis was induced (Bauer et al., 1999). However, co-expression of cyclophilin D or creatine kinase together with ANT I abolished apoptosis induction. Moreover, it has been observed that patients suffering from dilated cardiomyopathy had significantly higher ANT I messenger RNA levels (Dörner et al., 1997). This phenomenon, such 
as in the tissue culture experiments mentioned above, may be responsible for the increased apoptosis rate observed in case of cardiomyopathy. Considering that the ANT I in the peripheral inner membrane would form a permeability transition pore, we assume that proteins interacting with ANT I, such as cyclophilin and the octamer of mitochondrial creatine kinase, would reduce the probability of pore opening and apoptosis induction. However, the chance of pore opening and permeability transition would increase, if the ratio between the ANT I and its regulatory proteins is changing either by induction of ANT I or by dissociation of the octamer of mitochondrial creatine kinase.

\section{CONCLUSION}

The first electron microscopic observation of the dynamic behaviour of contact sites (Knoll \& Brdiczka, 1983) reflected morphological perturbations in mitochondrial membranes induced by association or dissociation of the described kinase complexes. The different components of the complexes interact depending on their actual structures that are regulated by the mitochondrial membrane potential and the energy metabolism of the cell. Physiologically, the complexes are transient structures that are subjected to regulation. In the isolated state they are stabilised because alternatively interacting partners have been removed. Thus the reconstituted complexes represent certain functional states that the different components have adopted. We have shown how perturbation of a single component, such as ANT, changes the function of the whole complex. This means that physiological signals will change the complex architecture as well, if they affect the properties of individual components, such as VDAC conductance or hexokinase or creatine kinases activity, with significant consequences for the cell.

\section{R E F E R E N C E S}

Adams V, Bosch W, Schlegel J, Wallimann T, Brdiczka D. (1989) Further characterization of contact sites from mitochondria of different tissues: topology of peripheral kinases. Biochim Biophys Acta.; 981: 213-25.

Bauer MK, Schubert A, Rocks O, Grimm S. (1999) Adenine nucleotide translocase-1, a component of the permeability transition pore, can dominantly induce apoptosis. $J$ Cell Biol.; 147: 1493-1502.

Benz R. (1994) Permeation of hydrophilic solutes through mitochondrial outer membranes: review on mitochondrial porins. Biochim Biophys Acta.; 1197: 167-96.

Benz R, Wojtczak L, Bosch W, Brdiczka D. (1988) Inhibition of adenine nucleotide transport through the mitochondrial porin by a synthetic polyanion. FEBS Lett.; 210: 75-80.

Benz R, Kottke M, Brdiczka D. (1990) The cationically selective state of the mitochondrial outer membrane pore: a study with intact mitochondria and reconstituted mitochondrial porin. Biochim Biophys Acta.; 1022: 311-8.

Beutner G, Rück A, Riede B, Welte W, Brdiczka D. (1996) Complexes between kinases, mitochondrial porin and adenylate translocator in rat brain resemble the permeability transition pore. FEBS Lett.; 396: 189-95.

Beutner G, Rück A, Riede B, Brdiczka D. (1998) Complexes between porin, hexokinase, mitochondrial creatine kinase and adenylate translocator display properties of the permeability transition pore. Implication for regulation of permeability transition by the kinases. Biochim Biophys Acta.; 1368: 7-18.

Beyer K, Klingenberg M. (1985) ADP/ATP carrier protein from beef heart mitochondria has high amounts of tightly bound cardiolipin, as revealed by 31P nuclear magnetic resonance. Biochemistry.; 24: 3821-6

Brdiczka D, Kaldis P, Wallimann T. (1994) In vitro complex formation between octamer of creatine kinase and porin. $J$ Biol Chem.; 269: 27640-4. 
Brustovetsky N, Klingenberg M. (1996) The mitochondrial ADP/ATP carrier can be reversibly converted into a large channel by $\mathrm{Ca}^{2+}$. Biochemistry.; 35: 8483-8.

Bücheler K, Adams V, Brdiczka D. (1991) Localization of the ATP/ADP translocator in the inner membrane and regulation of contact sites between mitochondrial envelope membranes by ADP. A study on freeze fractured isolated liver mitochondria. Biochim Biophys Acta.; 1056: 233-42.

Bühler S, Michels J, Wendt S, Rück A, Brdiczka D, Welte W, Przybylski M. (1998) Mass spectrometric mapping of ion channel proteins (Porins) and identification of their supramolecular membrane assembly. Proteins.; 2: 63-73.

Capano M, Crompton M. (2002) Biphasic translocation of Bax to mitochondria. Biochem J.; 367: 169-78.

Casadio R, Jacoboni I, Messina A, De Pinto V. (2002) A 3D model of the voltage-dependent anion channel (VDAC). FEBS Lett.; 520: 1-7.

Colombini M. (1979) A candidate for the permeability pathway of the outer mitochondrial membrane. Nature.; 279: 643-5.

Crompton M, Costi A. (1988) Kinetic evidence for a heart mitochondrial pore activated by $\mathrm{Ca}^{2+}$ and oxidative stress. Eur J Biochem.; 178: 448-501.

Crompton M, Ellinger H, Costi A. (1988) Inhibition by cyclosporin $\mathrm{A}$ of a $\mathrm{Ca}^{2+}$-dependent pore in heart mitochondria activated by inorganic phosphate and oxidative stress.

Biochem J.; 255: 357-60.

Crompton M, Virji S, Ward JM. (1998)

Cyclophilin-D binds strongly to complexes of the voltage-dependent anion channel and the adenine nucleotide translocase to form the permeability transition pore. Eur J Biochem.; 258: 729-35.

Dierks T, Salentin A, Heberger C, Krämer R. (1990) The mitochondrial aspartate/glutamate and ADP/ATP carrier switch from obligate counterexchange to unidirectional transport after modification by SH-reagents. Biochim Biophys Acta.; 1028: 268-80.
Dolder M, Wendt S, Wallimann T. (2001) Mitochondrial creatine kinase in contact sites: interaction with porin and adenine nucleotide translocase, role in permeability transition and sensitivity to oxidative damage. Biol Signals Recept.; 10: 93-111.

Dolder M, Walzel B, Spee O, Schlattner U, Wallimann T. (2003) Inhibition of the mitochondrial permeability transition by creatine kinase substrates, requirement for microcompartmentation. J Biol Chem.; 278: 17760-6.

Dörner A, Schulze K, Rauch U, Schultheiss HP. (1997) Adenine nucleotide translocator in dilated cardiomyopathy: pathophysiological alterations in expression and function. $\mathrm{Mol}$ Cell Biochem.; 174: 261-9.

Fiek C, Benz R, Roos N, Brdiczka D. (1982) Evidence for identity between the hexokinase-binding protein and the mitochondrial porin in the outer membrane of rat liver mitochondria. Biochim Biophys Acta.; 688: $429-40$.

Gellerich FN, Wagner M, Kapischke M, Wicker U, Brdiczka D. (1993) Effect of macromolecules on the regulation of the mitochondrial outer membrane pore and the activity of adenylate kinase in the inter-membrane space. Biochim Biophys Acta.; 1142: 217-27.

Gellerich FN, Laterveer FD, Zierz S, Nicolay K. (2002) The quantification of ADP diffusion gradients across the outer membrane of heart mitochondria in the presence of macromolecules. Biochim Biophys Acta.; 1554: $48-56$.

Gottlob K, Majewski N, Kennedy S, Kandel E, Robey RB, Hay N. (2001) Inhibition of early apoptotic events by Akt/PKB is dependent on the first committed step of glycolysis and mitochondrial hexokinase. Genes Dev.; 15: 1406-18.

Halestrap AP, Davidson AM. (1990) Inhibition of $\mathrm{Ca}^{2+}$-induced large amplitude swelling of mitochondria by cyclosporin $\mathrm{A}$ is probably caused by binding to a matrix peptidylprolyl cis-trans-isomerase and preventing it interact- 
ing with the adenine nucleotide translocase. Biochem J.; 268: 153-60.

Halestrap AP, McStay GP, Clarke SJ. (2002) The permeability transition pore complex: another view. Biochimie.; 84: 153-66.

Haworth RA, Hunter PR. (1980) Allosteric inhibition of the $\mathrm{Ca}^{2+}$-activated hydrophilic channel of the mitochondrial inner membrane by nucleotides J Membr Biol.; 57: 231-6.

Hashimoto M, Wilson JE. (2000) Membrane potential-dependent conformational changes in mitochondrially bound hexokinase in brain. Arch Biochem Biophys.; 884: 163-73.

Heldt HW, Klingenberg M, Milovancev M. (1972) Differences between the ATP-ADP ratios in the mitochondrial matrix and in the extramitochondrial space. Eur J Biochem.; 30: $434-40$.

Kinally KW, Zorov DB, Antonenko YN, Snyder SH, McEnery MW, Tedeschi H. (1993) Mitochondrial diazepine receptor linked to inner membrane ion channels by nanomolar actions of ligand. Proc Natl Acad Sci U S A.; 90: 1374-8.

Klingenberg M, Grebe K, Falkner G. (1971) Interaction between the binding of ${ }^{35} \mathrm{~S}$-atractyloside and bongkrekic acid at mitochondrial membranes. FEBS Lett.; 16: 301-3.

Kluck RM, Green DR, Newmeyer DD. (1997) The release of cytochrome $c$ from mitochondria: a primary site for Bcl-regulation of apoptosis. Science.; 275: 1132-6.

Knoll G, Brdiczka D. (1983) Changes in freeze-fracture mitochondrial membranes correlated to their energetic state. Biochim Biophys Acta.; 733: 102-10.

König T, Kocsis B, Mészaros L, Nahm K, Zoltán S, Horváth I. (1977) Interaction of a synthetic polyanion with rat liver mitochondria. Biochim Biophys Acta.; 462: 380-9.

Kottke M, Wallimann T, Brdiczka D. (1994) Dual localization of mitochondrial creatine kinase in brain mitochondria. Biochem Med Metabol Biol.; 51: 105-17.
Krämer R, Palmieri F. (1989) Molecular aspects of isolated and reconstituted carrier proteins from animal mitochondria. Biochim Biophys Acta.; 974: 1-23.

Lemeshko VV. (2002) Model of the outer membrane potential generation by the inner membrane of mitochondria. Biophys J.; 82: 684-92.

Liu X, Kim CN, Yang J, Jemmerson R, Wang X. (1996) Induction of apoptotic program in cell-free extracts: requirement for dATP and cytochrome c. Cell.; 86: 147-57.

Liu MY, Colombini M. (1992) A soluble mitochondrial protein increases the voltage dependence of the mitochondrial channel, VDAC. J Bioenerg Biomembr.; 24: 41-6.

O'Gorman E, Beutner G, Dolder M, Koretsky AP, Brdiczka D, Wallimann T. (1997) The role of creatine kinase in inhibition of mitochondrial permeability transition. FEBS Lett.; 414: $253-7$.

Ohlendieck K, Riesinger I, Adams V, Krause J, Brdiczka D. (1986) Enrichment and biochemical characterization of boundary membrane contact sites in rat-liver mitochondria.

Biochim Biophys Acta.; 860: 672-89.

Östlund AK, Göhring U, Krause J, Brdiczka D. (1983) The binding of glycerol kinase to the outer membrane of rat liver mitochondria: Its importance in metabolic regulation. Biochem Med.; 30: 231-45.

Pastorino JG, Tafani M, Rothman RJ, Marcinkeviciute A, Hoek JB, Farber JL. (1999) Functional consequences of sustained or transient activation by Bax of the mitochondrial permeability transition pore. $J$ Biol Chem.; 274: 31734-39.

Pastorino JG, Shulga N, Hoek JB. (2002) Mitochondrial binding of hexokinase II inhibits Bax-induced cytochrome $c$ release and apoptosis. J Biol Chem.; 277: 7610-18.

Rojo M, Hovius R, Demel RA, Nicolay K, Wallimann T. (1991) Mitochondrial creatine kinase mediates contact formation between mitochondrial membranes. J Biol Chem.; 266: 20290-5. 
Rostovtseva T, Colombini M. (1997) VDAC channels mediate and gate the flow of ATP: implications for the regulation of mitochondrial function. Biophys J.; 72: 1954-62.

Rück A, Dolder M, Wallimann T, Brdiczka D. (1998) Reconstituted adenine nucleotide translocase forms a channel for small molecules comparable to the mitochondrial permeability transition pore. FEBS Lett.; 426: $97-101$.

Saks V, Kuznetsov A, Khuchua Z, Vasilyeva E, Belikova J, Kesvatera T, Tiivel T. (1995) Control of cellular respiration in vivo by mitochondrial outer membrane and by creatine kinase. A new speculative hypothesis: possible involvement of mitochondrial-cytoskeleton interactions. J Mol Cell Cardiol.; 27: 625-45.

Saks V, Kaambre T, Sikk P, Eimre M, Orlova E, Paju K, Piirsoo A, Appaix F, Kay L, Regnitz-Zagrosek V, Fleck E, Seppet E. (2001) Intracellular energetic units in red muscle cells. Biochem J.; 356: 643-57.

Saleh ASS, Acharya S, Fishel R, Alnemri ES. (1999) Cytochrome $c$ and dATP-mediated oligomerization of Apaf- 1 is a prerequisite for procaspase-9 activation. $J$ Biol Chem.; 274: 17941-5.

Sampson MJ, Lovell RS, Craigen WJ. (1997) The murine voltage-dependent anion channel gene family. Conserved structure and function. J Biol Chem.; 272: 18966-73.

Schlattner U, Dolder M, Wallimann T, Tokarska-Schlattner M. (2001) Mitochondrial creatine kinase and mitochondrial outer membrane porin show direct interaction that is modulated by calcium. $J$ Biol Chem.; 276: 48027-30.

Soboll S, Brdiczka D, Jahnke D, Schmidt A, Schlattner U, Wendt S, Wyss M, Wallimann T. (1999) Octamer-dimer transitions of mitochondrial creatine kinase in heart disease. $J$ Mol Cell Cardiol.; 31: 857-66.

Song J, Midson C, Blachly-Dyson E, Forte M, Colombini M. (1998) The sensor regions of VDAC are translocated from within the membrane to the surface during the gating processes. Biophys J.; 74: 2926-44.
Vander Heiden MG, Chandel NS, Williamson EK, Schumacker PT, Thompson CB. (1997) Bcl-xL regulates the membrane potential and volume homeostasis of mitochondria. Cell.; 91: $627-37$.

Vyssokikh MY, Katz A, Rück A, Wünsch C, Dörner A, Zorov DB, Brdiczka D. (2001) Adenine nucleotide translocator isoforms 1 and 2 are differently distributed in the mitochondrial inner membrane and have distinct affinities to cyclophilin D. Biochem J.; 358: 349-58.

Vyssokikh MY, Zorova L, Zorov D, Heimlich G, Jürgensmeier JM, Brdiczka D. (2002) Bax releases cytochrome $c$ preferentially from a complex between porin and adenine nucleotide translocator. Hexokinase activity suppresses this effect. Mol Biol Rep.; 29: 93-6.

Weiler U, Riesinger I, Knoll G, Brdiczka D. (1985) The regulation of mitochondrial-bound hexokinases in the liver. Biochem Medicine.; 33: $223-35$

Welte W, Nestel U, Wacker T, Diederichs K. (1995) Structure and function of the porin channel. Kidney Int.; 48: 930-40.

Wicker U, Bücheler K, Gellerich FN, Wagner M, Kapischke M, Brdiczka D. (1993) Effect of macromolecules on the structure of the mitochondrial inter-membrane space and the regulation of hexokinase. Biochim Biophys Acta.; 1142: 228-39.

Więckowski MR, Vyssokikh M, Dymkowska D, Antonsson B, Brdiczka D, Wojtczak L. (2001) Oligomeric C-terminal truncated Bax preferentially releases cytochrome $c$ but not adenylate kinase from mitochondria, outer membrane vesicles and proteoliposomes. FEBS Lett.; 505: 453-9.

Xie G, Wilson JE. (1990) Tetrameric structure of mitochondrially bound rat brain hexokinase: A crosslinking study. Arch Biochem Biophys.; 276: 285-93.

Yang J, Liu X, Bhalla K, Kim CN, Ibrado AM, Cai J, Peng TI, Jones DP, Wang X. (1997) Prevention of apoptosis by Bcl-2: release of cytochrome $c$ from mitochondria blocked. Science.; 275: 1129-32. 
Zimmerberg J, Parsegian VA. (1986) Polymer inaccessible volume changes during opening and closing of a voltage-dependent ionic channel. Nature.; 323: 36-9.
Zoratti M, Szabó I. (1995) The mitochondrial permeability transition. Biochim Biophys Acta.; 1241: 139-76. 